\title{
Inter-plane Coupling and Spin Gap - an NMR/NQR Look on Typical Properties of High-Temperature Superconductors*
}

\author{
D. Brinkmann \\ Physik-Institut, University of Zürich, Winterthurer Strasse 190, CH-8057 Zürich, Switzerland
}

Z. Naturforsch. 51 a, 786-792 (1996); received November 20, 1995

\begin{abstract}
The paper discusses some NQR/NMR studies performed on $\mathrm{Y}-\mathrm{Ba}-\mathrm{Cu}-\mathrm{O}$ superconductors at the University of Zürich. In particular, we review studies performed in $\mathrm{Y}_{2} \mathrm{Ba}_{4} \mathrm{Cu}_{7} \mathrm{O}_{15}$ by measuring various planar $\mathrm{Cu} N Q R / N M R$ parameters: the spin-lattice relaxation time, the Knight shift and the indirect component of the Gaussian contribution to the spin-spin relaxation time. The temperature dependence of these parameters reveals a coupling between adjacent planes of a double plane. The existence of the inter-plane coupling has independently been confirmed by performing NQR SpinEcho Double Resonance (SEDOR) experiments. The appearance of a spin gap seems to be the consequence of inter-plane coupling.
\end{abstract}

Key words: NQR, NMR, High-Temperature Superconductors, Inter-plane Coupling, Spin gap.

\section{Introduction}

Up to now, an impressive number of nuclear magnetic resonance (NMR) and nuclear quadrupole resonance (NQR) experiments on planar copper and oxygen nuclei in cuprate high-temperature superconductors has been performed to elucidate the electronic spin dynamics of the $\mathrm{CuO}_{2}$ planes where superconductivity takes place. In most of these works, however, the aspect of the inter-plane coupling and its influence on the spin dynamics has not been addressed, even though neutron scattering experiments in $\mathrm{Y}-\mathrm{Ba}-\mathrm{Cu}-\mathrm{O}$ structures [1] revealed strong antiferromagnetic (AF) correlations between spins in different planes of the double plane. However, at elevated temperatures magnetic neutron scattering becomes extremely weak in the normal conducting state of $\mathrm{YBa}_{2} \mathrm{Cu}_{3} \mathrm{O}_{x}$, in particular when $x$ is close to 7 . Therefore, an alternative study of the inter-plane coupling in this temperature region by NMR/NQR could be of great help, especially with regard to the possible connection between inter-plane coupling and the spin pseudo-gap.

Such NMR/NQR studies became feasible with the synthesis of $\mathrm{Y}_{2} \mathrm{Ba}_{4} \mathrm{Cu}_{7} \mathrm{O}_{15}\left(T_{\mathrm{c}}=93 \mathrm{~K}\right)$, whose structure consists of a sequence of alternating $\mathrm{YBa}_{2} \mathrm{Cu}_{3} \mathrm{O}_{7}$

\footnotetext{
* Presented at the XIIIth International Symposium on Nuclear Quadrupole Interactions, Providence, Rhode Island, USA, July 23-28, 1995.
}

Reprint requests to Prof. D. Brinkmann.
(1-2-3 for short) and $\mathrm{YBa}_{2} \mathrm{Cu}_{4} \mathrm{O}_{8}(1-2-4)$ blocks containing single and double $\mathrm{CuO}$ chains, respectively. As in $\mathrm{YBa}_{2} \mathrm{Cu}_{3} \mathrm{O}_{7}$ and $\mathrm{YBa}_{2} \mathrm{Cu}_{4} \mathrm{O}_{8}$, the $\mathrm{CuO}_{2}$ planes in $\mathrm{Y}_{2} \mathrm{Ba}_{4} \mathrm{Cu}_{7} \mathrm{O}_{15}$ form double planes separated by $\mathrm{Y}$ ions. However, due to the alternation of 1-2-3 and 1-2-4 blocks the individual planes of a $\mathrm{CuO}_{2}$ double plane in $\mathrm{Y}_{2} \mathrm{Ba}_{4} \mathrm{Cu}_{7} \mathrm{O}_{15}$ are inequivalent and hence can separately be monitored by NMR/NQR methods allowing one to investigate the coupling between these planes. We will report on such studies performed in our laboratory.

\section{Probing the Dynamical Electron Susceptibility}

The electronic spin fluctuations in the $\mathrm{Y}-\mathrm{Ba}-\mathrm{Cu}-\mathrm{O}$ superconductors play an essential role. The lowfrequency spectrum of these fluctuations can be studied by probing the wave-vector $(q)$ and frequency $(\omega)$ dependent spin susceptibility, $\chi(q, \omega)$. Relevant information can be gained from the temperature dependence of three parameters: the magnetic shift tensor, the nuclear spin-lattice relaxation time, $T_{1}$, and the Gaussian contribution, $T_{2 \mathrm{G}}$, to the spin-spin relaxation time [2-4]. We briefly summarize how these parameters are related to $\chi(q, \omega)$.

The static part of the local magnetic hyperfine field gives rise to an NMR line shift expressed by the magnetic shift tensor $\mathbf{K}$, whose components, in an $x, y, z$ reference frame, can be decomposed in a spin and an 
orbital part:

$$
K_{\alpha \alpha}(T)=K_{\alpha \alpha}^{\mathrm{spin}}(T)+K_{\alpha \alpha}^{\text {orb }} .
$$

The $x, y, z$ reference frame quite often coincides with the principal axes system of the electric field gradient (EFG) tensor, $\mathbf{V}$, at the nuclear site. The spin part of the magnetic shift is usually called the Knight shift. In the high- $T_{\mathrm{c}}$ compounds, $\mathbf{K}^{\text {orb }}$ is predominantly temperature independent, whereas the temperature dependent $\mathbf{K}^{\text {spin }}$ is expected to vanish in the superconducting state due to singlet spin pairing.

Each part of the $\mathbf{K}$ tensor can be expressed by the respective hyperfine interaction tensor and the static electronic susceptibility as

$$
\begin{aligned}
K_{\alpha \alpha}^{\text {spin }} & =\frac{1}{g \mu_{\mathrm{B}}} \sum_{j}\left(A_{j}\right)_{\alpha \alpha}\left(\chi_{j}\right)_{\alpha \alpha}, \\
K_{\alpha \alpha}^{\text {orb }} & =\frac{1}{\mu_{\mathrm{B}}} O_{\alpha \alpha} \chi_{\alpha \alpha}^{\text {orb }} .
\end{aligned}
$$

The fluctuating part of the local hyperfine field is the source of the nuclear spin-lattice relaxation. In case of high- $T_{\mathrm{c}}$ compounds, the main contribution to the copper spin-lattice relaxation stems from the electron spin fluctuations. After Moriya [5], this contribution is related to the imaginary part of $\chi(q, \omega)$ in the following way:

$$
\begin{aligned}
& \left(\frac{1}{T_{1} T}\right)_{\alpha}=\frac{\gamma_{n}^{2} k_{\mathrm{B}}}{2 \mu_{\mathrm{B}}^{2}} \sum_{q, \alpha^{\prime} \neq \alpha}\left|A(q)_{\alpha^{\prime} \alpha^{\prime}}\right|^{2} \frac{\chi_{\alpha^{\prime} \alpha^{\prime}}^{\prime \prime}\left(\mathrm{q}, \omega_{0}\right)}{\omega_{0}}, \\
& A(q)_{\alpha \alpha}=\sum_{j} A_{j, \alpha \alpha} \exp \left(i \mathbf{q} \cdot \mathbf{r}_{j}\right) .
\end{aligned}
$$

Here, $\omega_{0}$ is the nuclear resonance frequency. $\alpha$ denotes the direction of quantization, i.e. the direction of $V_{z z}$ in NQR and of $\mathbf{B}_{0}$ (the external magnetic field) in NMR experiments, and $\alpha^{\prime}$ is the direction perpendicular to $\alpha$. $\mathbf{A}_{j}$ is the on-site $\left(\mathbf{r}_{j}=0\right)$ and the transferred $\left(\mathbf{r}_{j} \neq 0\right)$ hyperfine coupling tensor for the nuclei under consideration. Thus, the "relaxation rate per temperature unit" provides information about the $q$ averaged imaginary part of $\chi\left(q, \omega_{0}\right)$.

Pennington et al. [6] were the first to realize that the spin-spin relaxation rate of plane $\mathrm{Cu}$ in $\mathrm{YBa}_{2} \mathrm{Cu}_{3} \mathrm{O}_{7}$ is much larger than expected from conventional nuclear dipolar coupling. They showed that the predominant part of the rate is due to an enhanced $\mathrm{Cu}$ nuclear-nuclear spin coupling induced through an indirect coupling via electron spins. If the quantization axis of the $\mathrm{Cu}$ nuclear spin is parallel to the crystallographic $c$ axis, as in the case of plane copper in pure NQR, then
$T_{2 \mathrm{G} \text {, ind }}^{-1}$ can be expressed $[7,8]$ in terms of $\chi^{\prime}(q)$ as

$$
\begin{aligned}
{\left[\frac{1}{T_{2 \mathrm{G}, \text { ind }}}\right]^{2}=} & \frac{P}{m \hbar^{2}}\left[\frac{1}{N} \sum_{q}\left|A(q)_{c c}\right|^{4} \chi^{\prime}(q)^{2}\right. \\
& \left.-\left[\frac{1}{N} \sum_{q}\left|A(q)_{c c}\right|^{2} \chi^{\prime}(q)\right]^{2}\right] .
\end{aligned}
$$

Here, $P$ is the abundance of the $\mathrm{Cu}$ isotope being studied, $m$ is a constant that depends on the resonance method used ( $m=8$ for NMR and 4 for NQR), $N$ is the number of $\mathrm{Cu}$ atoms per unit area, and $c$ denotes the direction of quantization, i.e. the direction of the main component of the EFG tensor in case of NQR. A $(q)$ is again the Fourier transform of the hyperfine coupling tensor $\mathbf{A}\left(r_{i}\right)$ consisting of the on-site $A_{c c}\left(\mathbf{r}_{j}=0\right)$ and isotropic transferred $B\left(\mathbf{r}_{j} \neq 0\right)$ terms. For the $\mathrm{Cu}$ nuclei under consideration, and adopting the Mila-Rice Hamiltonian [9], $A(q)_{c c}$ is given by

$$
A(q)_{c c}=A_{c c}+2 B\left[\cos \left(q_{x} a\right)+\cos \left(q_{y} a\right)\right] .
$$

Since in all $\mathrm{Y}-\mathrm{Ba}-\mathrm{Cu}-\mathrm{O}$ compounds the spin part of the plane $\mathrm{Cu}$ magnetic shift in $c$ direction is zero, $A_{c c}=-4 B$. Consequently, $\mathbf{A}(q)$ peaks at the corners of the first Brillouin zone at the AF wave-vector $Q_{\mathrm{AF}}=\left( \pm \frac{\pi}{a}, \pm \frac{\pi}{a}\right)$, and $T_{2 \mathrm{G} \text {, ind }}^{-1}$ therefore involves predominantly $q$ summation of $\chi^{\prime}(q)$ close to $Q_{\mathrm{AF}}$.

\section{Measurement of Knight Shift and Relaxation in $\mathrm{Y}_{2} \mathrm{Ba}_{4} \mathrm{Cu}_{7} \mathrm{O}_{15}$}

We are now going to present the results of our $\mathrm{NMR} / \mathrm{NQR}$ measurements in $\mathrm{Y}_{2} \mathrm{Ba}_{4} \mathrm{Cu}_{7} \mathrm{O}_{15}$.

\subsection{Knight shift}

Details of the evaluation of the Knight shift from the $\mathrm{Cu}$ NMR spectrum were given in [10]. For $\mathbf{B}_{0} \perp \mathbf{c}$, we were able to extract, from the $\mathrm{Cu}$ spectrum, the temperature dependence of the total shift $K_{a a}\left(=K_{b b}\right)$ for $\mathrm{Cu}(2)$ of the 1-2-3 block and $\mathrm{Cu}(3)$ of the 1-2-4 block. However, to obtain the more relevant temperature dependent spin part $\mathbf{K}^{\text {spin }}$ one has to know the constant orbital part $\mathbf{K}^{\text {orb }}$, usually obtained as the rest $\mathbf{K}$ shift at temperatures far below $T_{c}$. In case of $\mathrm{Y}_{2} \mathrm{Ba}_{4} \mathrm{Cu}_{7} \mathrm{O}_{15}$, the broadening and the partial overlap of the two $\mathrm{Cu}$ lines prevents an accurate enough determination of $\mathbf{K}^{\text {orb }}$. Therefore we had to resort on the values obtained by Barret et al. for $\mathrm{YBa}_{2} \mathrm{Cu}_{3} \mathrm{O}_{7}$ [11] and by Zimmermann et al. for $\mathrm{YBa}_{2} \mathrm{Cu}_{4} \mathrm{O}_{8}$ [12]. 


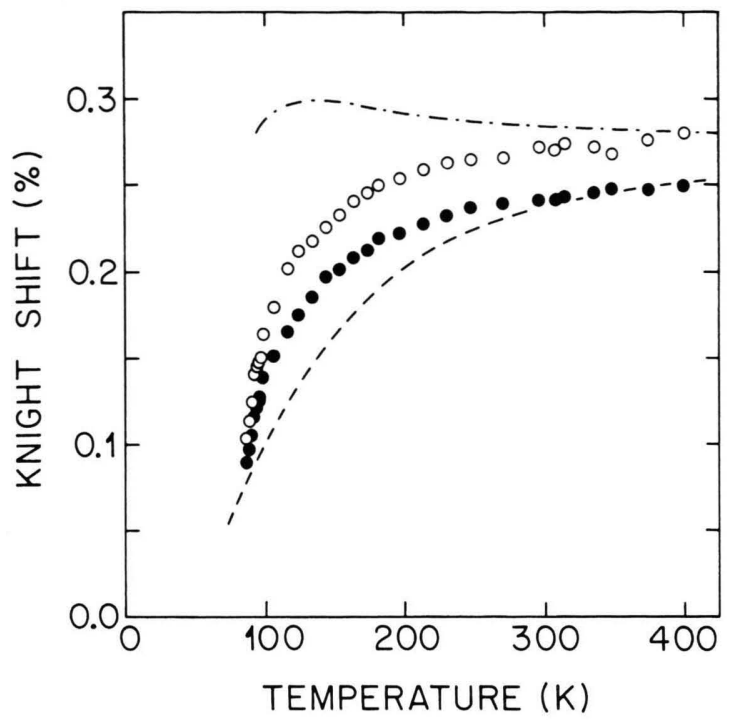

Fig. 1. Temperature dependence of the planar ${ }^{63} \mathrm{Cu}$ Knight shift at $\mathrm{Cu}(2)$ (open circles) and $\mathrm{Cu}(3)$ sites (filled circles) in $\mathrm{Y}_{2} \mathrm{Ba}_{4} \mathrm{Cu}_{7} \mathrm{O}_{15}$ [10] compared to the Knight shift at $\mathrm{Cu}(2)$ sites in $\mathrm{YBa}_{2} \mathrm{Cu}_{3} \mathrm{O}_{7}$ (dash-dotted line, [25] and in $\mathrm{YBa}_{2} \mathrm{Cu}_{4} \mathrm{O}_{8}$ (dashed line, [12]). The external field $\mathbf{B}_{0}$ lies perpendicular to the crystal $\mathbf{c}$ axis.

As one may expect both values agree in error limits. Thus we took the average value of $0.285 \%$ for $K_{a a}^{\mathrm{orb}}\left(=K_{b b}^{\mathrm{orb}}\right)$ of $\mathrm{Cu}(2)$ and $\mathrm{Cu}(3)$. Subtraction of $K_{a a}^{\mathrm{orb}}$ from the respective total $K_{a a}$ then yields the temperature dependent $K_{a a}^{\text {spin }}$ for $\mathrm{Cu}(2)$ and $\mathrm{Cu}(3)$ (see Figure 1).

\subsection{Spin-lattice relaxation}

The spin-lattice relaxation rate has been measured by NQR for all four copper sites of $\mathrm{Y}_{2} \mathrm{Ba}_{4} \mathrm{Cu}_{7} \mathrm{O}_{15}$. Figure 2 depicts the temperature dependence of $\left(T_{1} T\right)^{-1}$ of $\mathrm{Cu}(2)$ and $\mathrm{Cu}(3)$ together with related data from $\mathrm{YBa}_{2} \mathrm{Cu}_{3} \mathrm{O}_{7}$ and $\mathrm{YBa}_{2} \mathrm{Cu}_{4} \mathrm{O}_{8}$. Characteristic for $\mathrm{Y}_{2} \mathrm{Ba}_{4} \mathrm{Cu}_{7} \mathrm{O}_{15}$ is that $\left(T_{2} T\right)^{-1}$ for both planar sites depends strongly on temperature in the normal conducting state showing a maximum at $T^{*}=130 \mathrm{~K} \mathrm{com}$ bined with a Curie-Weiss type behavior above and a strong drop below $T^{*}$.

After the discovery of the spin-gap effect [13] in $\mathrm{YBa}_{2} \mathrm{Cu}_{3} \mathrm{O}_{7-\delta}$ by neutron scattering [14] several NMR groups have regarded the spin-gap effect to be responsible for the peculiar temperature variation of the relaxation rate, at least in the normal conducting state [4]. The occurrence of a spin gap means that

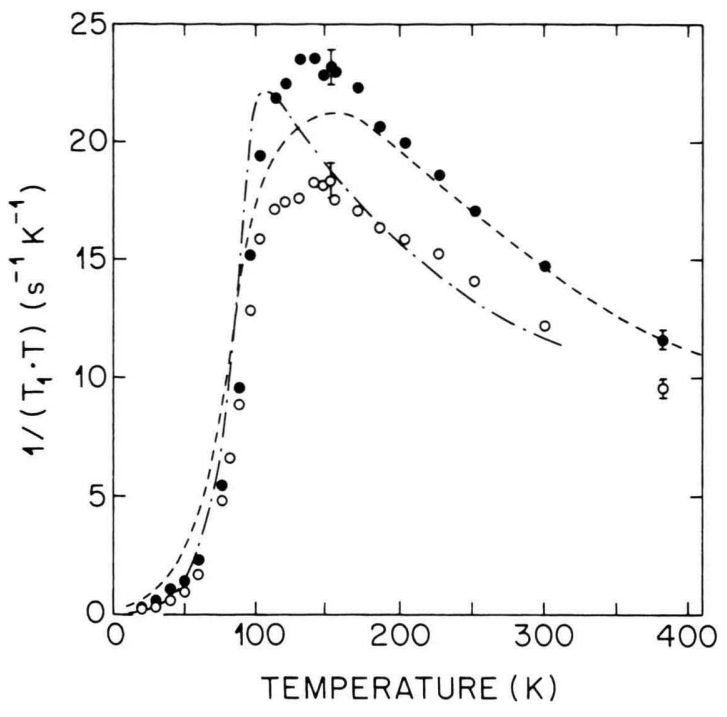

Fig. 2. Temperature dependence of ${ }^{63} \mathrm{Cu}\left(T_{1} T\right)^{-1}$ measured by $\mathrm{NQR}$ at $\mathrm{Cu}(2)$ (open circles) and $\mathrm{Cu}(3)$ sites (filled circles) in $\mathrm{Y}_{2} \mathrm{Ba}_{4} \mathrm{Cu}_{7} \mathrm{O}_{15}$ [10] compared to $\left(T_{1} T\right)^{-1}$ at $\mathrm{Cu}(2)$ sites in $\mathrm{YBa}_{2} \mathrm{Cu}_{3} \mathrm{O}_{7}$ (dash-dotted line, [26]) and in $\mathrm{YBa}_{2} \mathrm{Cu}_{4} \mathrm{O}_{8}$ (dashed line, [27]).

spectral weight in the electron spin fluctuations is transferred from lower to higher energy. The presence of the spin gap manifests itself in a maximum of $1 / T_{1} T$ at a temperature $T^{*}$ well above $T_{c}$. Therefore, the relaxation rate is described by an ad hoc formula:

$$
\frac{1}{T_{1} T}=\left(\frac{A_{0}}{T}\right)^{\alpha}\left[1-\tanh ^{2}\left(\frac{\Delta_{\mathrm{AF}}}{2 T}\right)\right] .
$$

Here, $\Delta_{\mathrm{AF}}$ denotes the spin-gap energy at $Q_{\mathrm{AF}}$ since the $\mathrm{Cu}$ relaxation is dominated by the strong AF fluctuations around $Q_{\mathrm{AF}} . A_{0}$ is a constant and the factor $T^{-\alpha}$ guarantees a reasonable description for the high-temperature behavior and may by attributed to the gradual decay of AF correlations at higher temperatures.

\subsection{Spin-spin relaxation}

The evaluation of $T_{2 \mathrm{G}}$ and hence of $T_{2 \mathrm{G} \text {,ind }}$ is more involved [15]. $T_{2 \mathrm{G}}$ follows from the decay of the spinecho amplitude, $E(2 \tau)$, recorded as a function of time $\tau$ between the first and the second pulse:

$$
E(2 \tau)=E_{0} \exp \left[-\frac{2 \tau}{T_{2 \mathrm{R}}}-\frac{1}{2}\left(\frac{2 \tau}{T_{2 \mathrm{G}}}\right)^{2}\right]
$$


where the Lorentzian-Redfield term $T_{2 \mathrm{R}}^{-1}$ stands for the decay rate due to the spin-lattice relaxation process. Details how to determine $T_{2 \mathrm{R}}^{-1}$ can be found in [15].

The obtained $T_{2 \mathrm{G}}^{-1}$ encompasses the temperature independent contribution, $T_{2 \mathrm{G} \text {,dip }}^{-1}$, arising from the direct nuclear dipole-dipole interaction and the temperature dependent contribution, $T_{2 \mathrm{G}}^{-1}$,ind , caused by the indirect nuclear spin-spin coupling mediated through the AF correlated electron spins. In a Gaussian approximation, neglecting the interference terms, both contributions add as [18]

$$
T_{2 \mathrm{G}}^{-2} \approx T_{2 \mathrm{G}, \mathrm{dip}}^{-2}+T_{2 \mathrm{G}, \text { ind }}^{-2} .
$$

Anticipating a common temperature dependence of $T_{2 \mathrm{G} \text {,ind }}^{-1}$ in both planes of $\mathrm{Y}_{2} \mathrm{Ba}_{4} \mathrm{Cu}_{7} \mathrm{O}_{15}$, we plotted $\left(T_{2 \mathrm{G}}^{-2}\right)_{124}$ vs. $\left(T_{2 \mathrm{G}}^{-2}\right)_{123}$ using the temperature as an implicit parameter. Within the experimental scatter a linear relationship $\left(T_{2 \mathrm{G}}^{-2}\right)_{124}=A\left(T_{2 \mathrm{G}}^{-2}\right)_{123}+B$ is indeed observed.

Figure 3 displays our results for $T_{2 \mathrm{G} \text {,ind }}$ in $\mathrm{Y}_{2} \mathrm{Ba}_{4} \mathrm{Cu}_{7} \mathrm{O}_{15}, \mathrm{YBa}_{2} \mathrm{Cu}_{3} \mathrm{O}_{6.982}$ and $\mathrm{YBa}_{2} \mathrm{Cu}_{4} \mathrm{O}_{8}$. For comparison, recent data for $\mathrm{YBa}_{2} \mathrm{Cu}_{3} \mathrm{O}_{6.9}$ [17], $\mathrm{Yba}_{2} \mathrm{Cu}_{3} \mathrm{O}_{6.98}$ [18] and $\mathrm{YBa}_{2} \mathrm{Cu}_{4} \mathrm{O}_{8}$ [16] are given. While there is rather good agreement for the $\mathrm{YBa}_{2} \mathrm{Cu}_{4} \mathrm{O}_{8}$ structures, there is a discrepancy for the $\mathrm{YBa}_{2} \mathrm{Cu}_{3} \mathrm{O}_{x}$ samples either for experimental reasons or because of a dependence of $T_{2 \mathrm{G}}$ on doping.

\section{Inter-plane Coupling in $\mathrm{Y}_{2} \mathrm{Ba}_{4} \mathrm{Cu}_{7} \mathrm{O}_{15}$}

The most important result of the previous section is summarized in Figure 4. It shows the temperature dependence of three ratios of NMR/NQR parameters of the two inequivalent planes in $\mathrm{Y}_{2} \mathrm{Ba}_{4} \mathrm{Cu}_{7} \mathrm{O}_{15}$ : (i) Knight shifts, $r_{\mathrm{K}}=K_{a a}^{\text {spin }}[\mathrm{Cu}(3)] / K_{a a}^{\text {spin }}[\mathrm{Cu}(2)]$; (ii) spin-lattice relaxation rates, $r_{W}=W[\mathrm{Cu}(3)] /$ $W[\mathrm{Cu}(2)]$; (iii) the indirect part to the Gaussian contribution to the spin-spin relaxation rate, $r_{T 2}=T_{2 \mathrm{G}, \text { ind }}^{-1}[\mathrm{Cu}(3)] / T_{2 \mathrm{G} \text {,ind }}^{-1}[\mathrm{Cu}(2)]$.

Above $100 \mathrm{~K}$, all three ratios are constant, the ratio $r_{T 2}$ is constant even above $14 \mathrm{~K}$. According to (2), (4) and (5), the temperature independent ratios imply a temperature independent relationship between the respective static and dynamic electronic spin susceptibilities. The relaxation rates, strictly speaking, involve $q$ average of $\chi\left(q, \omega_{0}\right)$. However, if we assume that the spin fluctuations at the corner of the Brillioun zone, $q=Q_{\mathrm{AF}}$, dominate the relaxation, the sum over $q$ can

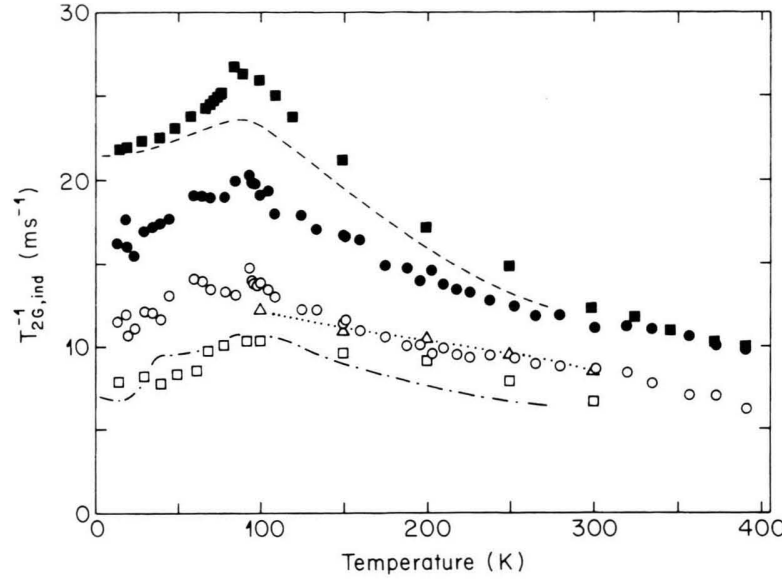

Fig. 3. Temperature dependence of NQR $T_{2 \mathrm{G} \text {,ind }}^{-1}$ at the planar copper sites in the $1-2-3$ (०) and $1-2-4$ blocks (๑) of $\mathrm{Y}_{2} \mathrm{Ba}_{4} \mathrm{Cu}_{7} \mathrm{O}_{15}$, in $\mathrm{YBa}_{2} \mathrm{Cu}_{3} \mathrm{O}_{6.982}(\square)$, and $\mathrm{YBa}_{2} \mathrm{Cu}_{4} \mathrm{O}_{8}(\square)$ [15]. For comparison, data for $\mathrm{YBa}_{2} \mathrm{Cu}_{3} \mathrm{O}_{6.9}(\Delta$, joined with dotted line, [17]), $\mathrm{YBa}_{2} \mathrm{Cu}_{3} \mathrm{O}_{6.98}$ (dash-dotted line, [18]), and $\mathrm{YBa}_{2} \mathrm{Cu}_{4} \mathrm{O}_{8}$ (dashed line, [16]).

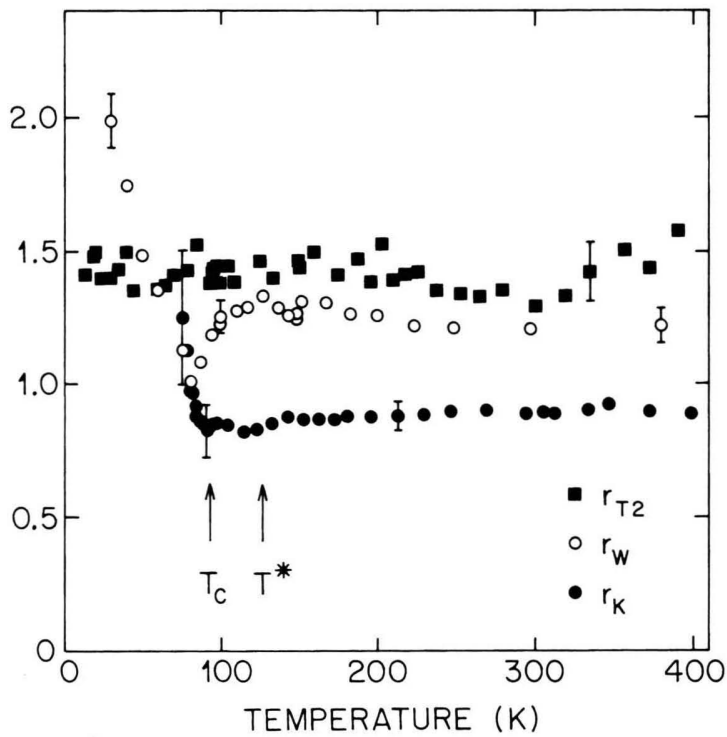

Fig. 4. Temperature dependence of the ratio of $\mathrm{Cu}(3)$ and $\mathrm{Cu}(2) \mathrm{NMR} / \mathrm{NQR}$ parameters in $\mathrm{Y}_{2} \mathrm{Ba}_{4} \mathrm{Cu}_{7} \mathrm{O}_{15}$ : Knight shifts (filled circles), spin-lattice relaxation rates (empty circles) and indirect components of the Gaussian contribution to the spin-spin relaxation rate (squares).

be replaced by $\chi^{\prime \prime}\left(Q_{\mathrm{AF}}\right)$ and $\chi^{\prime}\left(Q_{\mathrm{AF}}\right)$, respectively. The plane copper hyperfine constants appearing in the ratios are alike in $\mathrm{Y}-\mathrm{Ba}-\mathrm{Cu}-\mathrm{O}$ compounds $[19,20]$ and seem to be temperature independent. In other words: both the static and the dynamic electron susceptibili- 
ties of the two single planes of each double-plane are governed by the same temperature dependence which means common spin dynamics in both planes, and hence these planes must be strongly coupled. However, since $r_{\mathrm{K}}, r_{W}$ and $r_{T 2}$ are not equal, the $q$ dependence of the susceptibility is not the same in the two planes.

The coupling strength may be estimated from the $K_{a a}^{\text {spin }}$-vs.-temperature plot (Figure 1). Below $300 \mathrm{~K}$, the $K_{a a}^{\text {spin }}$ shifts in the 1-2-3 and 1-2-4 blocks begin to depart from those in $\mathrm{YBa}_{2} \mathrm{Cu}_{3} \mathrm{O}_{7}$ and $\mathrm{YBa}_{2} \mathrm{Cu}_{4} \mathrm{O}_{8}$ due to the interplane coupling, thus the coupling strength is not much less than $k_{\mathrm{B}} \cdot 300 \mathrm{~K}$ or $30 \mathrm{meV}$.

Why is the common temperature dependence of the Knight shift and of the spin-lattice relaxation rate lost below $T_{c}$ ? A loss of the inter-plane coupling is unlikely. A possibility could be the formation of two superconducting gaps that differ in the individual planes because of their different charge carrier densities, $n$. However, up to now even the spatial symmetry of the superconducting state in $\mathrm{Y}-\mathrm{Ba}-\mathrm{Cu}-\mathrm{O}$, and so the form of the superconducting gap is not well established, let alone its dependence on $n$. Thus, the explanation of the temperature variation of $r_{W}$ and $r_{\mathrm{K}}$ below $T_{c}$ is still missing.

\section{SEDOR experiments in $\mathrm{Y}_{2} \mathrm{Ba}_{4} \mathrm{Cu}_{7} \mathrm{O}_{15}$}

We have performed NQR spin-echo double resonance (SEDOR) experiments in $\mathrm{Y}_{2} \mathrm{Ba}_{4} \mathrm{Cu}_{7} \mathrm{O}_{15}$ [21] to confirm independently and directly the existence of the inter-plane coupling and to determine the temperature dependence of the resulting inter-plane component of the static electron spin susceptibility in the normal state. To our knowledge, it is the first SEDOR experiment performed in $\mathrm{Y}_{2} \mathrm{Ba}_{4} \mathrm{Cu}_{7} \mathrm{O}_{15}$ and the first in high- $T_{c}$ superconductors involving nuclei in different planes. Observing a SEDOR proves that the two nuclei involved are coupled by a spin-spin interaction [22].

The SEDOR experiment proceeds as follows. We perform a spin-echo experiment on nuclear spin $I$, e.g. the $\mathrm{Cu}(2)$ spin, by applying a $\pi / 2-\tau-\pi$ radio-frequency pulse sequence at the NQR frequency of $I$ and observing the echo of the $I$ spins; $\tau$ denotes the time separation of the two pulses. In addition we apply, at time $\tau_{\mathrm{F}}$ after the $\pi / 2$ pulse, another $\pi$ pulse at the frequency of spin $S$, e.g. the $\mathrm{Cu}(3)$ spin. This pulse flips the $S$ spin and thus changes the local field produced by

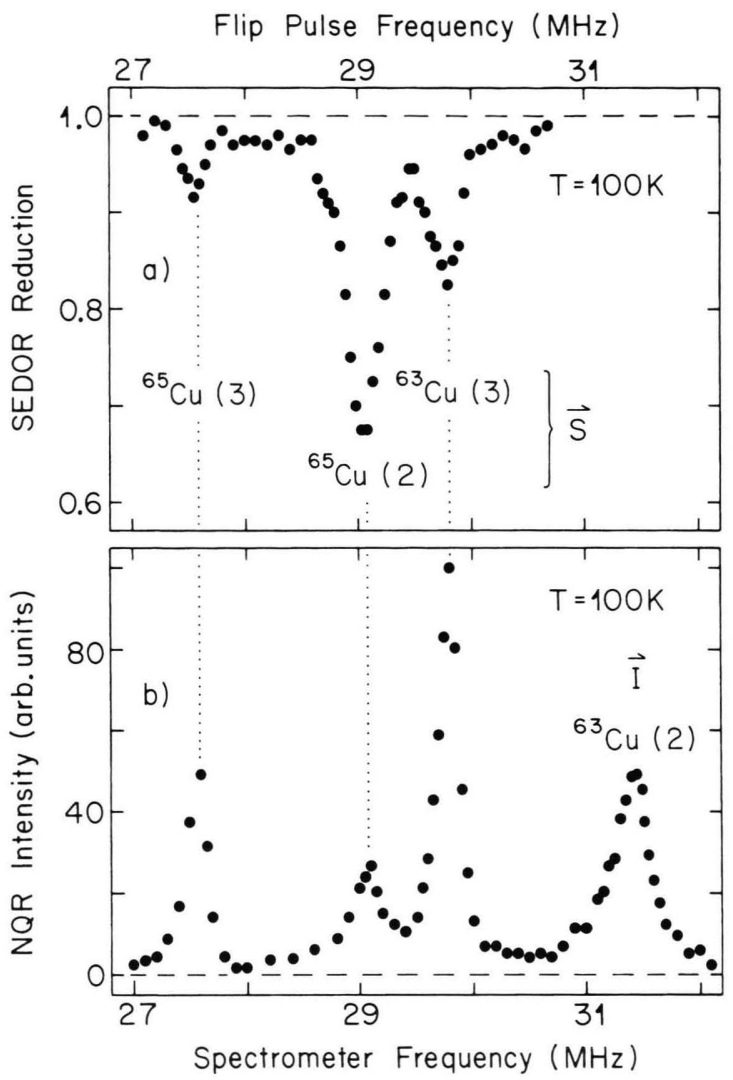

Fig. 5. SEDOR spectrum (a) and NQR spectrum (b) of planar $\mathrm{Cu}$ sites in $\mathrm{Y}_{2} \mathrm{Ba}_{4} \mathrm{Cu}_{7} \mathrm{O}_{15}$ [21]. The spin-echo of the ${ }^{63} \mathrm{Cu}(2)$ nucleus is observed. The vertical dotted lines indicate the NQR frequency of the $S$ spin with which $I$ interacts for a particular flip pulse frequency.

$S$ at $I$. Hence the echo formation of $I$ is disturbed, resulting in a reduction of the echo. We call this an $I-S$ SEDOR experiment. By comparing the $I$ spin echo with and without the $S$ spin flipping one can deduce the coupling between $I$ and $S$ spins. Details of the experiments are given in [21].

Figure 5 serves as an example. The lower part shows the planar $\mathrm{Cu}$ NQR spectrum of $\mathrm{Y}_{2} \mathrm{Ba}_{4} \mathrm{Cu}_{7} \mathrm{O}_{15}$ powder at $100 \mathrm{~K}$. The upper part displays the reduction of the $I$ spin-echo in an $I-S$ SEDOR with variable $S$ spin flip frequency. In this case, $I$ is the ${ }^{63} \mathrm{Cu}(2)$ nucleus and its echo reduction has been measured by sweeping the flip frequency from 27 to $30.5 \mathrm{MHz}$ with both $\tau$ and $\tau_{\mathrm{F}}$ kept constant. The $I$ echo is reduced whenever the flip frequency coincides (as indicated by vertical dotted lines) with one of the NQR frequencies of the other signals. The SEDOR reduction thus re- 
veals the coupling of the ${ }^{63} \mathrm{Cu}(2)$ nucleus with both ${ }^{65} \mathrm{Cu}(2)$ of the same plane and ${ }^{63} \mathrm{Cu}(3)$ and ${ }^{65} \mathrm{Cu}(3)$ of the adjacent inequivalent plane. The time dependence of the echo amplitude, $E_{\mathrm{S}}\left(2 \tau_{\mathrm{F}}\right)$, can be described by a Gaussian:

$$
E_{\mathrm{S}}\left(2 \tau_{\mathrm{F}}\right)=E_{\mathrm{S} 0} \exp \left[-\frac{1}{2}\left(\frac{2 \tau_{\mathrm{F}}}{T_{\mathrm{S}}}\right)^{2}\right],
$$

where $T_{\mathrm{S}}$ denotes the SEDOR relaxatioin time.

Pennington et al. [6] showed that for intra-plane $\mathrm{Cu}$ SEDOR, $T_{\mathrm{S}}$ of the $I$ spin is related to the $S$ spin's time constant $T_{2 \mathrm{G}}$ by

$$
T_{\mathrm{S}}^{\text {intra }}(I)=\frac{\gamma(S)}{\gamma(I)} T_{2 \mathrm{G}}(S),
$$

where $\gamma$ is the respective gyromagnetic ratio. $T_{2 \mathrm{G}}$ is related to $T_{2 \mathrm{G} \text {,ind }}$ as discussed above.

By performing SEDOR experiments with various combinations of $\mathrm{Cu}$ nuclei within one plane and between both planes of a double plane we clearly demonstrated the existence of indirect spin-spin couplings in the plane and between planes. Since this coupling is mediated by exchange coupled electronic moments of planar $\mathrm{Cu}$ ions, the results are direct evidence for electronic inter-plane coupling.

In particular, we form the ratio of $T_{2 \mathrm{G} \text {,ind }}$ of the 1-2-4 block plane and $T_{\mathrm{S} \text {, ind }}$ of the 63(2)-63(3) SEDOR. This ratio can be considered as the ratio of the inter-plane and intra-plane components of the static electron spin susceptibility and a such reflects the strength of the inter-plane coupling (represented by $T_{\mathrm{S} \text {,ind }}^{-1}$ ) with respect to the intra-plane coupling (given by $T_{2 \mathrm{G} \text {,ind }}^{-1}$.

A first attempt to link $T_{2 \mathrm{G}, \text { ind }} / T_{\mathrm{S} \text {,ind }}$ to the interplane exchange coupling constant, $J_{\perp}$, has been made by Millis and Monien [23]. Using a simiplified model for the susceptibility of two $\mathrm{CuO}_{2}$ planes coupled by an inter-plane coupling $J_{\perp}$, the authors deduce from our experimental data the product of $J_{\perp}$ and the maximum value of the susceptibility, $\chi_{\max }$. At $120 \mathrm{~K}$, they

[1] J. M. Tranquada, P. M. Gehring, G. Shirane, S. Shamoto, and M. Sato, Phys. Rev. B. 46, 5561 (1992).

[2] C. H. Pennington and C. H. Slichter, in: Physical Properties of high temperature superconductors II, ed. D. M Ginsberg, World Scientific, New York 1990.

[3] Appl. Magn. Reson. 3, 383 (1992). A special issue containing contributions of NMR/NQR work in cuprate superconductors. get for this product 0.4 which, together with $\chi_{\max } \approx 80$ states/eV-Cu [7], would imply $J_{\perp} \approx 5 \mathrm{meV}$. Although small, this value appears to be of the right order of magnitude. Obviously, for a more reliable value, a more involved analysis is necessary.

\section{Relation Between Spin Gap and Inter-plane Coupling}

The origin of the spin gap is still under debate. For instance, one may ask whether the gap is an intrinsic property of the single $\mathrm{CuO}_{2}$ plane or a consequence of inter-plane effects.

Pursuing the first path, Sokol and Pines [24] propose a unified magnetic phase diagram of the cuprate superconductors, which, dependent on doping level and temperature, displays various regimes that, among others, are characterized by certain temperature independent ratios of plane $\mathrm{Cu} T_{1} T$ and $T_{2 \mathrm{G} \text {,ind }}$ values. In the quantum critical (QC) regime (applicable to spin gap compounds), the ratio $T_{1} T / T_{2 \mathrm{G} \text {,ind }}$ is constant while in the overdamped (OD) regime $T_{1} T / T_{2 \mathrm{G} \text {,ind }}^{2}$ is constant.

We have checked these predictions [15] and found that $\mathrm{YBa}_{2} \mathrm{Cu}_{3} \mathrm{O}_{7}$ is in the OD regime from $T_{c}$ up to $300 \mathrm{~K}$, and that the underdoped $\mathrm{YBa}_{2} \mathrm{Cu}_{4} \mathrm{O}_{8}$ structure is in the QC regime for temperatures above $T^{*}$. However, $\mathrm{Y}_{2} \mathrm{Ba}_{4} \mathrm{Cu}_{7} \mathrm{O}_{15}$ does not fit into this scheme. Instead, taking the inter-plane coupling as the origin of the spin gap, quite naturally accounts for the observed $\mathrm{Y}_{2} \mathrm{Ba}_{4} \mathrm{Cu}_{7} \mathrm{O}_{15}$ behavior. The inter-plane coupling not only dictates a common temperature dependence of the dynamic susceptibility in adjacent planes, it also leads to a common value of the spin gap in the fluctuation spectrum. Alhough we cannot demonstrate that the inter-plane coupling is indeed responsible for the occurrence of the spin gap, our results point to the importance of the interplane coupling in the spin gap formation.

[4] D. Brinkmann and M. Mali, in: NMR - Basic Principles and Progress, ed. by P. Diehl, E. Fluck, H. Günther, R. Kosfeld, J. Seelig, vol. 31, p. 171, Springer, Berlin 1994.

[5] T. Moriya, J. Phys. Soc. Japan 18, 516 (1963).

[6] C. H. Pennington, D. J. Durand, C. P. Slichter, J. P. Rice. E. D. Bukowski, and D. M. Ginsberg, Phys. Rev. B. 39, 274 (1989). 
[7] C. H. Pennington and C. P. Slichter, Phys. Rev. Lett. 66, 381 (1991)

[8] (a) D. Thelen and D. Pines, Phys. Rev. B. 49, 3528 (1994); (b) M. Takigawa, ibid., 4158 (1994).

[9] F. Mila and T. M. Rice, Physica C 157, 561 (1989).

[10] R. Stern, M. Mali, I. Mangelschots, J. Roos, D. Brinkmann, J.-Y. Genoud, T. Graf, and J. Müller, Phys. Rev. B. 50, 426 (1994).

[11] S. E. Barrett, D. J. Durand, C. H. Pennington, C. P. Slichter, T. A. Friedmann, J. P. Rice, and D. M. Ginsberg, Phys. Rev. B. 41, 6283 (1990).

[12] H. Zimmermann, M. Mali, I. Mangelschots, J. Roos, L. Pauli, D. Brinkmann, K. Karpinski, S. Rusiecki, and E. Kaldis, J. Less-Comm. Met. 164-165, 138 (1990).

[13] T. M. Rice, in: The physics and chemistry of oxide superconductors, edited by Y. Iye, Springer 1992.

[14] J. Rossat-Mignod, L. P. Regnault, C. Vettier, P. Burlet, J. Y. Henry, and G. Lapertot, Physica B 169, 58 (1991).

[15] R. Stern, M. Mali, J. Roos, and D. Brinkmann, Phys. Rev. B. 51, 15478 (1995).

[16] Y. Itoh, H. Yasuoka, Y. Fujiwara, Y. Ueda, T. Machi, I. Tomeno, K. Tai, N. Koshizuka, and S. Tanaka, J. Phys. Soc. Japan 61, 1287 (1992).
[17] T. Imai, C. P. Slichter, A. P. Paulikas, and B. Veal, Phys. Rev. B. 47, 9158 (1993); Appl. Magn. Reson. 3, 729 (1992).

[18] Y. Itoh, K. Yoshimura, T. Ohomura, H. Yasuoka, Y. Ueda, and K. Kosuge, J. Phys. Soc. Japan 63, 1455 (1994).

[19] T. Imai, J. Phys. Soc. Japan 59, 2508 (1990).

[20] H. Zimmermann, Ph. D. thesis, University of Zurich (1991).

[21] R. Stern, M. Mali, J. Roos, and D. Brinkmann, Phys. Rev. B. 52 (1 December 1995).

[22] C. P. Slichter, in: Nuclear Magnetic Double Resonance, edited by B. Maraviglia, North-Holland 1993, p. 69.

[23] A. Millis and H. Monien, to be published.

[24] A. Sokol and D. Pines, Phys. Rev. Lett. 71, 2813 (1993).

[25] R. E. Walstedt, R. F. Bell, L. F. Schneemeyer, J. V. Waszczak, and G. P. Espinosa, Phys. Rev. B. 45, 8074 (1992).

[26] M. Mali, D. Brinkmann, L. Pauli, J. Roos, H. Zimmermann, and J. Hulliger, Phys. Lett A 124, 112 (1987).

[27] H. Zimmermann, M. Mali, D. Brinkmann, J. Karpinski, E. Kaldis, and S. Rusiecki, Physica C 159, 681 (1989). 